\title{
Ground-state Shallow-donor Binding Energy in (In,Ga)N/GaN Double QWs Under Temperature, Size, and the Impurity Position Effects
}

\author{
Redouane En-Nadir ${ }^{*}$, Haddou El Ghazi ${ }^{1,2}$, Anouar Jorio ${ }^{1}$, Izeddine Zorkani ${ }^{1}$ \\ ${ }^{1}$ LPS, FSDM, Sidi Mohammed Ben Abdellah University, Fes, Morocco \\ ${ }^{2}$ ENSAM Laboratory, ENSAM, Hassan II University, Casablanca, Morocco \\ * Corresponding author email: redouane.en-nadir@usmba.ac.ma \\ Received: 25 February 2021 / Accepted: 27 March 2021 / Published: 30 March 2021
}

\begin{abstract}
In this paper, we study the hydrogen-like donor-impurity binding energy of the ground-state change as a function of the well width under the effect of temperature, size, and impurity position. Within the framework of the effective mass approximation, the Schrodinger-Poisson equation has been solved taken account an on-center hydrogen-like impurity in double QWs with rectangular finite confinement potential profile for $10 \%$ of indium concentration in the (well region). The eigenvalues and their correspondent eigenvectors have been obtained by the fined element method (FEM). The obtained results are in good agreement with the literature and show that the temperature, size, and the impurity position have a significant impact on the binding energy of a hydrogen-like impurity in symmetric double coupled quantum wells based on non-polar wurtzite (In,Ga) N/GaN core/Shell.
\end{abstract}

Keywords: Binding energy, Finite confinement potential, double QWs, Temperature, and Impurity.

\section{Introduction}

For many years ago, the understanding of semiconductors was one of the most interested goals of researchers in the field of materials science particularly, the presence of impurity either donor or acceptor of electrons. Therefore, we need to understand the behavior of an electron in materials under various external and internal physical parameters such as temperature, pressure, magnetic and electric fields. Many studies show that both electrical and optical proprieties of inorganic semiconductor's nanostructures change under the presence of impurity. Furthermore, the carriers (electron, and hole) confinement in nanostructures such as quantum wells (QWs) [1], quantum dots (QDs) [2], and other geometrical-shapes with finite confinement potential has demonstrated fantastic proprieties for modern and future optoelectronics technology [3]- [4]- [5]. The binding energy of hydrogen-like impurity in nanostructured semiconductors made out with different band-gaps materials under various effects concerning different electronic-states has been reported in many works. H. El Ghazi, and coworkers have investigated the stark effect and pressure-dependent on ground-state donor binding energy in In $\mathrm{GaN} / \mathrm{GaN}$ square and parabolic QWWs [6] - [7]. The effect of a spatially dependent effective mass on the hydrogenic impurity binding energy in a finite parabolic quantum well has been reported by XH.Qi et al [8]. Sali. A and coworkers have investigated the combined effect of pressure and temperature on the impurity binding energy in a cubic quantum dot using the FEM simulation [9]. Başer, P, and coworkers have studied the hydrostatic pressure and temperature effects on hydrogenic impurity binding energies in GaAs/InxGa1-xAs/GaAs square quantum well [10]. The simultaneous effects of hydrostatic pressure and temperature on the binding energy of hydrogenic impurity in cylindrical quantum well wires have been reported by Karki and coworkers [11]. Recent works have reported the temperature-dependent of impurity binding energy. In 2018 El-Yadri et al. have investigated the temperature and hydrostatic pressure effects on single dopant states in hollow 
cylindrical core-shell quantum dot [12]. Dong and coworkers have investigated the binding energy and dynamics of charge-transfer states in organic photovoltaics with the low driving force for charge separation [13].

To the best of our knowledge, the lowest-state donor-impurity binding energy in double quantum wells (DQWs) with rectangular finite confinement potential considering $10 \%(v=0.1)$ of indium in (well region) has not been reported previously. In this paper, we examined the effect of temperature, size, and impurity position in the structure on the ground-state binding energy of a hydrogen-like impurity in GaN/InGaN double QWs using the fined element method.

\section{Theory and Calculation}

Using the fined element method (FEM) within the framework of the approximation effective-mass, the quasi-one-dimensional Schrödinger equation describing the behavior of a particle free to move along z-axe in a Nano-semiconductor taking account the presence of a hydrogen-like impurity located in $\lambda_{0}$, with considering a rectangular confinement potential profile is given as:

$$
\frac{-\hbar^{2}}{2} \frac{d}{d z}\left[\frac{1}{m_{e}^{*}(v, T)} \frac{d \psi_{n}(z)}{d z}\right]+\left(V_{0}(\mathrm{z}, v, \mathrm{~T})-\frac{e^{2}}{\varepsilon_{r}^{*}(v, T)\left|\vec{r}-\overrightarrow{r_{0}}\right|}\right) \psi_{n}(z)=E_{n} \psi_{n}(z)
$$

Where $V_{0}, e$ and $m_{e}^{*}$ are the confinement potential, the electron mass and the electron charge respectively. $\hbar$ is the Planck constant and $\varepsilon_{r}^{*}$ is the relative dielectric constant of the considered semiconductor, and $\left|\vec{r}-\overrightarrow{r_{0}}\right|$ is the impurity electron distance.

The confinement potential profile along the growth (z-axe) is modulated as following,

$$
V_{0}(\mathrm{z}, v, \mathrm{~T})=\left\{\begin{array}{l}
0, \quad\left\{\begin{array}{c}
L \leq z \leq L+l \\
L+l+W \leq z \leq L+W+2 l
\end{array}\right. \\
Q . \Delta E g(v) \quad, \quad \text { elsewhere }
\end{array}\right.
$$

$L, W$, and $l$ defines the outer-barriers, the intermediate-barrier, and the wells widths respectively.

Where $\mathrm{Q}(=0.7), \Delta \mathrm{E}_{g}^{I n G a N}(\nu, T)$ is the indium concentration-dependent, and the temperature difference energy between $\mathrm{GaN}$ and $\mathrm{InN}$ band-gaps is given as fellows,

$$
\Delta E_{g}^{\operatorname{InGaN}}(v, T)=v E_{g}^{\operatorname{InN}}(T)+(1-v) E_{g}^{G a N}(T)-b(1-v)
$$

The confinement potential energy $V_{0}(z, v, T)$, is related to the conduction-band offset energy of the $\mathrm{GaN} / \mathrm{InGaN}$ hetero-structure by the following expression,

$$
\mathrm{V}_{0}(v, T)=Q\left[E_{g}^{G a N}(v, T)-E_{g}^{I I G a N}(v, T)\right]
$$

The band-gaps energy temperature-dependent of the binaries $\mathrm{InN}$ and $\mathrm{GaN}$ are given as follows [14],

$$
E_{g}^{i}(T)=E_{g}^{i}(T=0 K)-\frac{\alpha \times 10^{-3} \times T^{2}}{T+\beta}
$$

The dielectric constants versus the temperature for both $\mathrm{GaN}$ and $\mathrm{InN}$ are given by the following approximated [14],

$$
\begin{aligned}
& \varepsilon_{G a N}^{*}(v, T)=\varepsilon_{G a N}^{*}(T=300 K) \exp \left((T-300) \times T^{-4}\right) \\
& \varepsilon_{\text {InGaN }}^{*}(v, T)=\varepsilon_{G a N}^{*}(v, T)+0.6 v
\end{aligned}
$$

According to the $\vec{K} . \vec{P}$ theory, the effective-mass temperature-dependent is given by the expression below [14]:

$$
\frac{m_{0}}{m_{i}^{*}(v, T)}=1+\frac{C_{i}}{E_{g}^{i}(v, T)} \quad i \equiv \operatorname{InN}, \text { GaN }
$$

$\mathrm{C}_{\mathrm{i}}$ is the energy related to the momentum matrix element. 
En-Nadir et al., J. Mod. Sim. Mater.; Vol. 4 Issue 1, pp: 1-6, 2021

The binding energy is defined as the difference of the energy between the energy without and with impurity respectively. It is given as:

$$
E_{b}=E_{0}-E_{I}
$$

Where $E_{0}$ and $E_{I}$ are respectively, the energy without, and with impurity.

\section{Results and Discussion}

In this paper, we have used the effective units, i.e, the effective Bhor radius $a^{*}(=2.69 \mathrm{~nm})$ as the unit of the width, and the effective Rydberg energy $R^{*}(=27.71 \mathrm{eV})$ as the unit of energy. Table.1 shows different physical parameters of $\mathrm{GaN}$ and $\mathrm{InN}[14]$

Table.1: Different physical parameters of GaN and InN [14].

\begin{tabular}{|c|c|c|}
\hline Parameters & GaN & InN \\
\hline $\boldsymbol{E}_{\boldsymbol{g}}^{\boldsymbol{i}}(\mathbf{T}=\mathbf{0})$ & 3.51 & 0.70 \\
\hline $\boldsymbol{m}_{\boldsymbol{i}}^{*}(\mathbf{T}=\mathbf{0})\left(\boldsymbol{m}_{\mathbf{0}}\right)$ & 0.19 & 0.04 \\
\hline $\boldsymbol{\varepsilon}_{\boldsymbol{i}}^{*}\left(\boldsymbol{\varepsilon}_{\mathbf{0}}\right)$ & 9.6 & 10.5 \\
\hline $\boldsymbol{C}_{\boldsymbol{i}}^{*}$ & 14.7 & 15.5 \\
\hline $\boldsymbol{\alpha}$ & 0.909 & 624 \\
\hline $\boldsymbol{\beta}$ & 0.245 & 830 \\
\hline
\end{tabular}

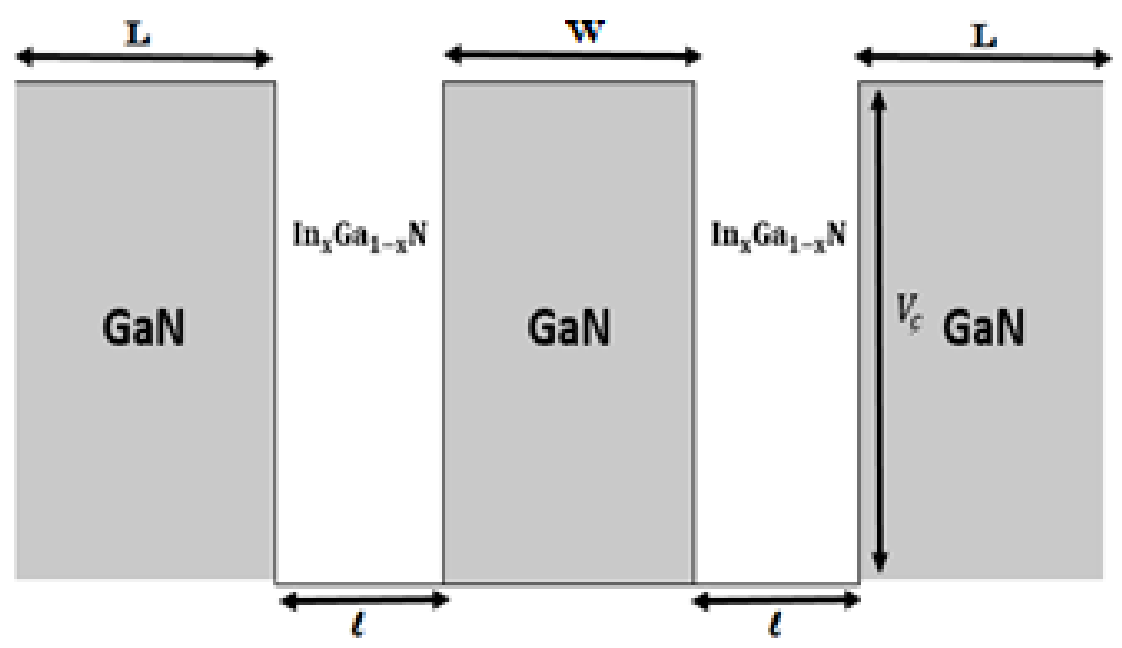

Figure 1: The schematic description of the unstrained $G a N /(I n, G a) N / G a N$ Double QWs, and the corresponding confinement potential profile.

Fig.1. Shows the schematic description of the unstrained $\mathrm{GaN} /(\mathrm{In}, \mathrm{Ga}) \mathrm{N} / \mathrm{GaN}$ core/shell double QWs. Also, shows the corresponding confinement potential profile in which we considered the active region (well) which is made out of $\mathrm{InGaN}$ alloy enveloped by $\mathrm{GaN}$ as the barrier semiconductor material, and we take account the continuity of the electronic wave function in the interfaces between InGaN, and $\mathrm{GaN}$ semiconductors materials. $l, W$, and $L$ represent the well, the coupling barrier, and the outer-barriers widths, respectively. 
(a)

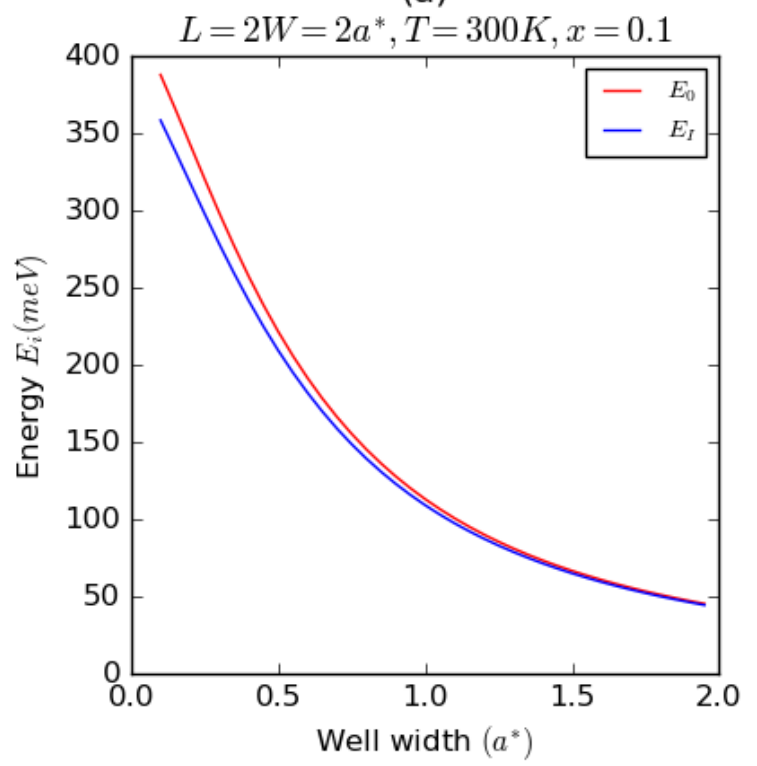

(b)

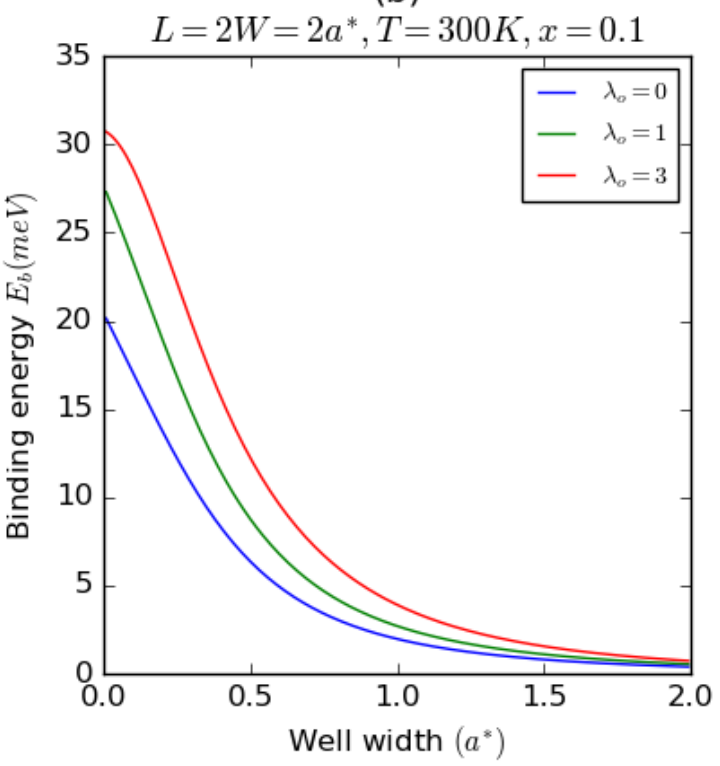

Figure 2: The variation of the energy (a), and the binding energy for three value of

impurity-position (b) as a function the well width.

In Fig.2. The variation of the electron-energy in the case without $\left(E_{0}\right)$, and with an impurity $\left(E_{I}\right)$ as a function of the well width is presented. Now considering the Fig.2. (a), we clearly see that the energy of both contributions without and with impurity decrease when the well width increase. This is due to the fact that the more the well width increases the more the confinement potential inside the well gets weaker and weaker, the electron starts to feel like a free-electron in a bulk semiconductor then the energy diminishes as well. Also, in the same figure, we noticed that the electron-energy in the case without is more important than the electron-energy with impurity particularly for a well width less than the effective Bhor radius $\left(a^{*}\right)$. This is can be explained by the fact that when the impurity term is added to the Hamiltonian as a perturbation, the electron-energy decreases because of the Colombian interaction between the electron and impurity. Furthermore, H. Bahramiyan, and coworker, have demonstrated that the binding energy is larger when the impurity locates at the position of maximum probability due to the reason that the stronger Columbic coupling provided that the electronic density of probability has its maximum located exactly at the impurity-position [15]. In the same context, W.Belaid and coworkers have proved that the impact of the impurity's position is more significant in DQW in particularly for the moderate confinement regime [6].

The binding energy variation versus the well with three values of the impurity-position is presented in Fig.2. (b). Firstly, we noticed that the binding energy decrease as well as the well width increase to reach a constant value for a well width more than $\left(1.5 a^{*}\right)$. This behavior is expected because when the well width increase, the confinement inside the well becomes weak, consequently the impurity binding energy decrease. Also, we observed that the closer the impurity to the center on the structure passing from $\left(\lambda_{0}: 0 \rightarrow 3\right)$ the more the binding energy decreases. Owning to the fact that the more the impurity goes towards the center the more the distance between the electron and the impurity decreases, consequently the binding energy increases in parallel. 
En-Nadir et al., J. Mod. Sim. Mater.; Vol. 4 Issue 1, pp: 1-6, 2021

(a)

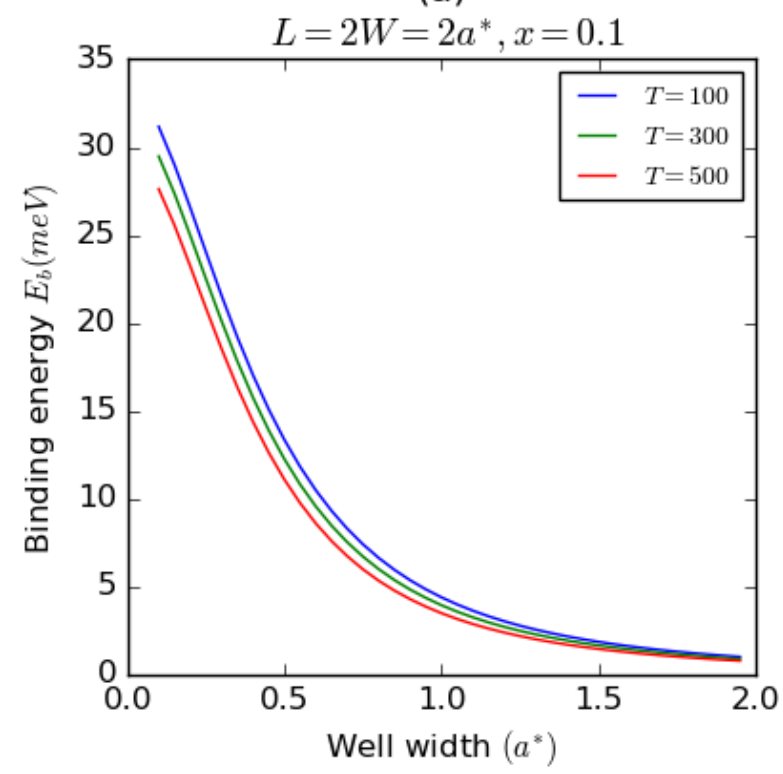

(b)

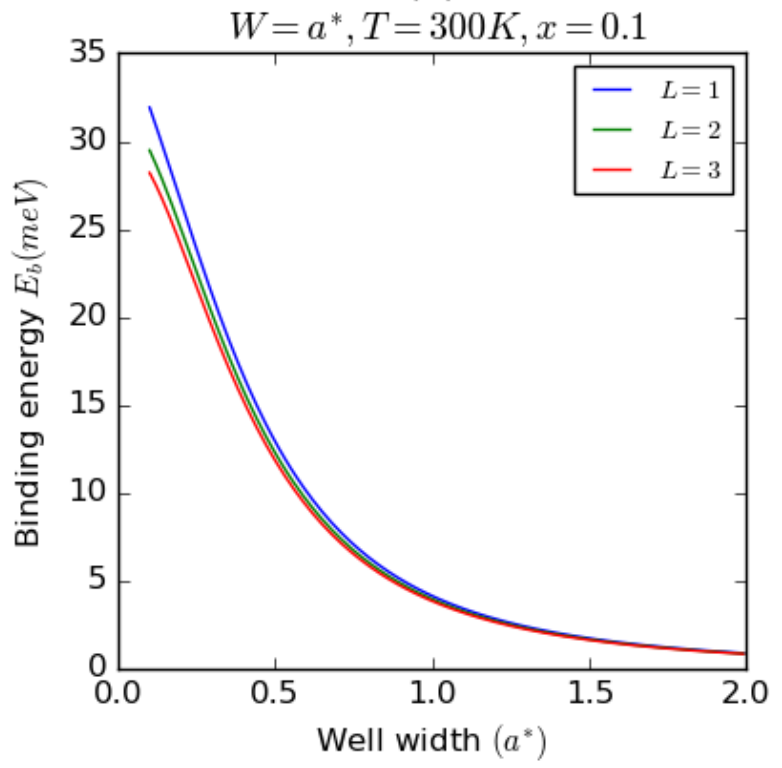

Figure 3: The variation of the binding as a function the well width, taken account the effect

of temperature (a), and the outer-barrier size (b).

In Figure.3. Shows the variation of the impurity binding energy as a function of the well width, the effect of temperature (a), and the outer-barrier-width (b) are presented. From fig.3.(a), we can see clearly that the impurity binding energy decrease as discussed before in Fig.2. to reach a stable value for all well widths above $\left(1.5 a^{*}\right)$ as well as it decreases when the temperature passes from $100 \mathrm{~K}$ to $500 \mathrm{~K}$. This behavior can physically be explained by the thermal energy concept. According to the thermal energy, the hydrogenicimpurity gains more kinetic energy, and the wave function is delocalized to a larger extent. Therefore, it becomes less bound with the donor-impurity atom. The same thing has been approved by Khordad and coworkers for the ground, and the lowest excited-state [16]. Additionally, A.Sali and H.Satori have reported that the increase of temperature leads to a reduction of the ground-state binding energy of a donor impurity and its effect is quite significant in small QDs only [9]. The effect of the outer-barrier width on the impurity binding energy has been examined in Fig.3 (b). We see clearly that the outer-barrier width is more significant in the strong confinement regime $\left(l<0.7 a^{*}\right)$. The binding energy decrease with the augmentation of the outer-barrier width $\left(L: 1 a^{*} \rightarrow 3 a^{*}\right)$. The physical reason that when the outer-barrier width increase the well width decreases for a fixed value of the intermediate-barrier width $\left(\mathrm{W}=a^{*}\right)$ and then the wave function starts to feel the barrier potential of the outer-barrier and then starts to penetrate into the barriers mage of $\mathrm{GaN}$ which lead an increase in the electron-impurity distance. Consequently, the binding energy decrease to reach a stable value in the regime of weak confinement $\left(l>a^{*}\right)$. The same thing has been reported by W.Blaid and coworkers in previous Ref. [6].

\section{Conclusion}

To sum up, we have investigated the binding energy of a hydrogen-like donor-impurity in non-polar Wurtzite symmetric double InGaN/GaN QWs using the fined element method. Our results are in good agreement with the works of literature. The obtained results showed that the temperature, size, and impurity-position have a remarkable impact on the impurity binding energy. Our results reveal that: The electron-energy in case without-impurity $\left(E_{0}\right)$ is more significant of that in case with-impurity $\left(E_{I}\right)$ in the strong confinement regime $\left(l<a^{*}\right)$. The $E_{b}$ increase when the impurity goes toward the center of the nanostructure. The $E_{b}$ decrease when the temperature increase and pass from $100 \mathrm{~K}$ to $500 \mathrm{~K}$. Also, the $E_{b}$ decrease when the outer-barrier width increase and pass from $a^{*}$ to $3 a^{*}$. 


\section{Competing Interests}

The authors declared that they have no conflict of interest in publishing this work.

\section{How to Cite this Article:}

R. En-nadir, H. El Ghazi, A. Jorio, and I. Zorkani, "Ground-state Shallow-donor Binding Energy in (In,Ga)N/GaN Double QWs Under Temperature, Size, and the Impurity Position Effects”, J. Mod. Sim. Mater., vol. 4, no. 1, pp. 1-6, Mar. 2021.

\section{References}

[1] N. Bochkareva, A. Ivanov, A. Klochkov and Y. Shreter, "Gaussian impurity bands in GaN and weakening of carrier confinement in InGaN/GaN quantum wells", Journal of Physics: Conference Series, vol. 1697, p. 012203, 2020. https://doi.org/10.1088/17426596/1697/1/012203

[2] C. Bose and C. Sarkar, "Effect of a parabolic potential on the impurity binding energy in spherical quantum dots", Physica B: Condensed Matter, vol. 253, no. 3-4, pp. 238-241, 1998. https://doi.org/10.1016/s0921-4526(98)00407-4

[3] Y. Li et al., "High-efficiency near-UV light-emitting diodes on Si substrates with InGaN/GaN/AlGaN/GaN multiple quantum wells", Journal of Materials Chemistry C, vol. 8, no. 3, pp. 883-888, 2020. https://doi.org/10.1039/c9tc06138j

[4] B. Su, H. He, H. Zhang, X. Pan and Z. Ye, "Photoluminescence properties of $\mathrm{ZnO} / \mathrm{ZnMgO}$ multiple quantum wells under high excitation", Superlattices and Microstructures, vol. 139, p. 106418, 2020. https://doi.org/10.1016/j.spmi.2020.106418

[5] K. Fellaoui, A. Oueriagli and D. Abouelaoualim, "Linear and nonlinear optical absorptions in III-V nitrides quantum well with semiparabolic confining potential", Indian Journal of Physics, vol. 93, no. 10, pp. 1353-1357, 2019. https://doi.org/10.1007/s12648-01901378-x

[6] W. Belaid, H. El Ghazi, I. Zorkani and A. Jorio, "Impact of QW coupling on the binding energy in InGaN/GaN under the effects of the size, the impurity and the internal composition", MATEC Web of Conferences, vol. 330, p. 01012, 2020. https://doi.org/10.1051/matecconf/202033001012

[7] H. Ghazi, A. Jorio and I. Zorkani, "Pressure-dependent shallow donor binding energy in InGaN/GaN square QWWs", Physica B: Condensed Matter, vol. 410, pp. 49-52, 2013. https://doi.org/10.1016/j.physb.2012.10.027

[8] X. Qi, X. Kong and J. Liu, "Effect of a spatially dependent effective mass on the hydrogenic impurity binding energy in a finite parabolic quantum well", Physical Review B, vol. 58, no. 16, pp. 10578-10582, 1998. https://doi.org/10.1103/physrevb.58.10578

[9] A. Sali and H. Satori, "The combined effect of pressure and temperature on the impurity binding energy in a cubic quantum dot using the FEM simulation", Superlattices and Microstructures, vol. 69, pp. 38-52, 2014. https://doi.org/10.1016/j.spmi.2014.01.011

[10] P. Başer, I. Altuntas and S. Elagoz, "The hydrostatic pressure and temperature effects on hydrogenic impurity binding energies in GaAs/InxGa1-xAs/GaAs square quantum well", Superlattices and Microstructures, vol. 92, pp. 210-216, 2016. https://doi.org/10.1016/j.spmi.2015.12.010

[11] H. Karki, S. Elagoz and P. Başer, "Simultaneous effects of hydrostatic pressure and temperature on the binding energy of hydrogenic impurity in cylindrical quantum well wires", Superlattices and Microstructures, vol. 48, no. 3, pp. 298-304, 2010. https://doi.org/10.1016/j.spmi.2010.06.021

[12] M. El-Yadri, N. Aghoutane, A. El Aouami, E. Feddi, F. Dujardin and C. Duque, "Temperature and hydrostatic pressure effects on single dopant states in hollow cylindrical core-shell quantum dot", Applied Surface Science, vol. 441, pp. 204-209, 2018. https://doi.org/10.1016/j.apsusc.2018.01.195

[13] Y. Dong et al., "The binding energy and dynamics of charge-transfer states in organic photovoltaics with low driving force for charge separation", The Journal of Chemical Physics, vol. 150, no. 10, p. 104704, 2019. https://doi.org/10.1063/1.5079285

[14] M. Karimi, G. Rezaei and M. Nazari, "Linear and nonlinear optical properties of multilayered spherical quantum dots: Effects of geometrical size, hydrogenic impurity, hydrostatic pressure and temperature", Journal of Luminescence, vol. 145, pp. 55-60, 2014. https://doi.org/10.1016/j.jlumin.2013.07.046

[15] H. Bahramiyan and R. Khordad, "Effect of various factors on binding energy of pyramid quantum dot: pressure, temperature and impurity position", Optical and Quantum Electronics, vol. 46, no. 5, pp. 719-729, 2013. https://doi.org/10.1007/s11082-013-9782-1

[16] R. Khordad, "Effect of temperature on the binding energy of excited states in a ridge quantum wire", Physica E: Low-dimensional Systems and Nanostructures, vol. 41, no. 4, pp. 543-547, 2009. https://doi.org/10.1016/j.physe.2008.10.004

\section{Publish your research article in AIJR journals- \\ $\checkmark \quad$ Online Submission and Tracking \\ $\checkmark$ Peer-Reviewed \\ $\checkmark$ Rapid decision \\ $\checkmark \quad$ Immediate Publication after acceptance \\ $\checkmark$ Articles freely available online \\ $\checkmark \quad$ Retain full copyright of your article.}

Submit your article at journals.aijr.org

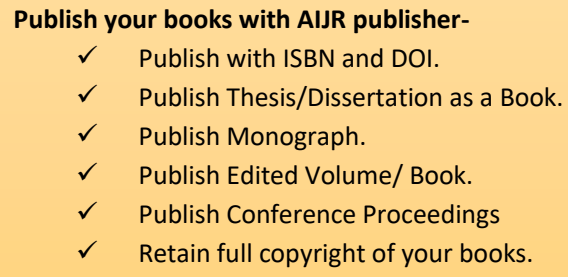

Submit your manuscript at books.aijr.org 